Vol.12. No. 10

Eur. J. Clin. Microbiol. Infect. Dis., October 1993, p. 750-755

0934-9723/93/10 0750-06 $\$ 3.00 / 0$

\title{
Evaluation of Penicillin G in the Prevention of Streptococcal Septicaemia in Patients with Acute Myeloid Leukaemia Undergoing Cytotoxic Chemotherapy
}

\author{
P. de Jong ${ }^{1}$, M. de Jong ${ }^{2}$, E. Kuijper ${ }^{3}$, J. van der Lelie ${ }^{1,2 *}$
}

\begin{abstract}
The efficacy of penicillin $G$ was evaluated in the prevention of infections caused by streptococci in patients receiving remission induction or intensive consolidation treatment for acute myeloid leukaemia. Between 1980 and 1988, 29 episodes of streptococcal septicaemia occurred in 139 treatment events. All patients received as prophylaxis regimen ciprofloxacin $(n=38)$ or a combination of polymyxin $B$ with nalidixic acid $(n=42)$ or neomycin $(n=59)$. Six patients died of streptococcal septicaemia despite adequate antibiotic treatment. The high incidence of streptococcal septicaemia lead to the administration of penicilin $\mathbf{G}$ in addition to ciprofloxacin as prophylaxis regimen during the 14 days immediately following cytotoxic chemotherapy. Only two episodes of streptococcal septicaemia were documented after addition of penicillin $G$ to the prophylaxis regimen $(n=76, p<0.001)$. Both patients had an uneventful recovery after treatment with vancomycin. Patients receiving penicillin $G$ prophylaxis experienced fever during $17 \%$ of the time and received antimicrobial therapy during $20 \%$ of the time per treatment event compared with $27 \%$ and $32 \%$ respectively of this time in patients receiving no streptococcal prophylaxis $(p<0.001)$. Penicillin $G$ prophylaxis was associated with an increased incidence of fever of unknown origin and more frequent isolation of aerobic gram-negative bacteria in surveillance cultures. Penicillin $G$ in combination with ciprofloxacin proved to be highly successful in preventing infections caused by streptococci and in reducing infection-related mortality and morbidity.
\end{abstract}

Several studies have shown an increased incidence of septicaemia due to streptococci in patients with acute leukaemia undergoing intensive cytotoxic chemotherapy (1-4). Although gramnegative bacteria used to be the predominant pathogens causing infection, streptococci are now a frequent cause of morbidity and mortality in these patients. Complications of streptococcal septicaemia include respiratory distress syndrome and septic shock (5-7). Mortality rates vary between $10 \%$ and $38 \%(1,8)$.

The change in the pathogen spectrum of bacterial infections and the severity of these infections have lead to revision of the strategies for management of neutropenic patients. Since currently used prophylactic regimens do not prevent streptococcal septicaemia, additional administration of vancomycin $(9,10)$, roxithromycin (11) or

\footnotetext{
${ }^{1}$ Department of Haematology, ${ }^{2}$ Department of Internal Medicine, and ${ }^{3}$ Department of Microbiology, Academic Medical Centre, University of Amsterdam, Amsterdam, The Netherlands.
}

penicillin (12) has been suggested. Other investigators recommend the routine empirical use of vancomycin (13) or penicillin $G(8)$ upon onset of fever. Heimdahl et al. (14) recommend limiting the portals of entry for streptococci by preventing oral herpes simplex virus infections.

As previously reported, we have also noticed an increasing incidence of streptococcal septicaemia in our centre (4). Therefore, since 1988 we have added penicillin $G$ to our ciprofloxacin prophylaxis regimen during the first 14 days following cytotoxic chemotherapy for acute myeloid leukaemia. In this article, we report the results using this extended regimen to prevent streptococcal infection and compare the results with our previous experience using prophylactic regimens not containing penicillin $\mathrm{G}$.

\section{Patients and Methods}

Clinical and microbiological records were reviewed of all 86 adult patients who were treated in our hospital for acute myeloid leukaemia between 1980 and 1990, and 
received either ciprofloxacin or a combination of polymyxin B with nalidixic acid or neomycin for selective intestinal decontamination. They received 236 courses of antileukaemic chemotherapy leading to moderate neutropenia $\left(<500\right.$ neutrophils $/ \mathrm{mm}^{3}$ ) or severe neutropenia $\left(<100\right.$ neutrophils $\left./ \mathrm{mm}^{3}\right)$. Besides dose increment, no essential changes were made in antileukaemic treatment during this period. A treatment event was defined as the period of time between the start of cytotoxic chemotherapy and either a rise of the neutrophil count above $500 / \mathrm{mm}^{3}$, or the start of a new course of cytotoxic chemotherapy, or discontinuation of active treatment, with a minimum of seven days. Twenty-one events were excluded from analysis because of insufficient data (12) or a duration of less than seven days (9). The evaluable 215 events included 148 remission inductions and 67 intensive consolidations.

Prophylactic antibiotics were administered orally from the start of cytotoxic chemotherapy until a rise of the neutrophil count above $500 / \mathrm{mm}^{3}$. The different prophylaxis regimens used consisted of polymyxin B $(200 \mathrm{mg}$ q.i.d.) in combination with either nalidixic acid ( $1 \mathrm{~g}$ q.i.d., 42 events, $1980-1982$ ) or neomycin ( $250 \mathrm{mg}$ q.i.d., 59 events 1982-1986), and ciprofloxacin ( $500 \mathrm{mg}$ b.i.d., 38 events, 1986-1988). In the last two years intravenous penicillin $\mathrm{G}$ ( $10^{6} \mathrm{U}$ q.i.d., 63 events) was given in addition to ciprofloxacin during the first 14 days following chemotherapy. Three patients were allergic to penicillin $\mathrm{G}$ and received as alternative prophylaxis erythromycin $(500 \mathrm{mg}$ q.i.d., 3 events), vancomycin ( $500 \mathrm{mg}$ q.i.d., 2 events) or cephradine ( $1 \mathrm{~g}$ q.i.d., 2 events). Five patients were treated for an early infection with vancomycin $(500 \mathrm{mg}$ q.i.d., 4 events) or cephradine (1 g q.i.d., 2 events) before the penicillin $G$ prophylaxis was started, and these drugs were continued as streptococcal prophylaxis. Patients also received antifungal prophylaxis (amphotericin $\mathrm{B}$, $500 \mathrm{mg}$ q.i.d., or ketoconazole, $200 \mathrm{mg}$ b.i.d.) and were nursed in simple reversed isolation when neutrophil counts were below $500 / \mathrm{mm}^{3}$.

A rebrile episode was defined as a temperature $>38^{\circ} \mathrm{C}$ of at least 12 hours duration or a temperature $>38.5^{\circ} \mathrm{C}$ on one occasion. In case of fever, patients were examined calefully for the presence of infection, and cultures of blood and material from suspected sites of infection were performed. Depending on the patient's condition, broadspectrum antibiotic therapy was instituted empirically, usually consisting of a combination of gentamicin and amoxicillin in patients not receiving penicillin $G$, and gentamicin and vancomycin in patients receiving penicillin G. Febrile episodes were classified afterwards as clinically documented infections (signs of infection, negative cultures), microbiologically documented infections (signs of infection, positive cultures of blood or other material) or fever of unknown origin (no signs of infection, negative cultures). Clinically documented infections were further classified as minor infections (rapidly resolving infeclions, mainly superficial skin infections) or major infections (prolonged and/or lifc-threatening infections, mainly lung infections). Septicaemia was diagnosed when signs of infection were present and one or more blood cultures were positive. In the case of septicaemia due to Staphylococcus epidermidis and Corynebacterium xerosis at least two positive blood cultures were required.

Surveillance cultures of specimens from the throat and faeces were performed twice weekly and were cultured for Staphylococcus aureus, aerobic gram-negative bac- teria, yeasts and fungi. From 1985 onwards cultures of nasal specimens were performed as well. Colonization was defined as the isolation of a microorganism from one or more surveillance cultures during a treatment event after at least one week of prophylaxis.

For statistical analysis the chi-square test and the Student's t-test were used. A $p$ value $\leq 0.05$ was considered to be statistically significant.

\section{Results}

Patients and Treatment Events. Data on the patients and treatment events are shown in Table 1. The median numbers of days of prophylaxis, moderate neutropenia and severe neutropenia were higher in patients receiving ciprofloxacin with or without streptococcal prophylaxis than in the other patient group, probably due to the use of more intensive cytotoxic chemotherapy in recent years. The median age of patients in the ciprofloxacin group was higher.

Fever and Antimicrobial Therapy. The data on febrile episodes and antimicrobial therapy are summarized in Table 1. Patients receiving streptococcal prophylaxis experienced fever and received antimicrobial therapy for a significantly shorter time per treatment event compared with patients receiving ciprofloxacin alone $(\mathrm{p}<0.001)$ or polymyxin $B$ combined with nalidixic acid or neomycin $(\mathrm{p}<0.001)$. Furthermore, the period of time between the start of cytotoxic chemotherapy and the first day of fever was significantly longer in patients receiving ciprofloxacin plus streptococcal prophylaxis compared with patients receiving either ciprofloxacin alone $(p=0.024)$ or polymyxin $B$ plus neomycin or nalidixic acid $(p=0.0004)$. The period of time between the start of cytotoxic chemotherapy and the first day of antimicrobial therapy in patients receiving ciprofloxacin plus streptococcal prophylaxis was not significantly different from that in patients receiving ciprofloxacin alone $(p=0.117)$, but was significantly shorter in patients receiving polymyxin $B$ plus nalidixic acid or neomycin $(p=0.022)$.

Febrile Episodes, Streptococcal Septicaemia and Infection-Related Mortality. The number and causes of febrile episodes for each prophylaxis group are shown in Table 2. All groups had comparable numbers of febrile episodes. In patients receiving ciprofloxacin plus streptococcal prophylaxis, 16 febrile episodes occurred before streptococcal prophylaxis was given, 36 during streptococcal prophylaxis and 13 after discon- 
Table 1: Data on patients, treatment events, prophylaxis and antimicrobial therapy.

\begin{tabular}{|c|c|c|c|}
\hline & $\begin{array}{l}\text { Polymyxin } \mathrm{B}+ \\
\text { neomycin or } \\
\text { nalidixic acid }\end{array}$ & Ciprofloxacin & $\begin{array}{l}\text { Ciprofloxacin + } \\
\text { streptococcal } \\
\text { prophylaxis }\end{array}$ \\
\hline No. of treatment events & 101 & 38 & 76 \\
\hline No. of patients & 48 & 18 & 28 \\
\hline Age (years) ${ }^{b}$ & $54(24-75)$ & $64.5(22-77)$ & $53(20-75)$ \\
\hline Duration (days) of prophylaxis ${ }^{b}$ & $20(7-51)$ & $25(7-39)$ & $26(9-83)$ \\
\hline Duration (days) of neutropenia ${ }^{b}$ & $16(0-51)$ & $21.5(6-39)$ & $20(0-74)$ \\
\hline Duration (days) of severe neutropeniab & $9(0-42)$ & $14.5(0-30)$ & $14(0-14)$ \\
\hline Infection at start of prophylaxis & 8 & 5 & 6 \\
\hline Duration (days) of fever & $552 / 2146(26 \%)$ & $250 / 924(27 \%)$ & $360 / 2109(17 \%)$ \\
\hline Time (days) until first day of fever ${ }^{b}$ & $6(0-19)$ & $7(0-21)$ & $11(0-29)$ \\
\hline Duration (days) of antimicrobial therapy & $698 / 2146(33 \%)$ & $297 / 924(32 \%)$ & $425 / 2109(20 \%)$ \\
\hline $\begin{array}{l}\text { Time (days) until first day of } \\
\text { antimicrobial therapyb }\end{array}$ & $8(0-23)$ & $11(0-21)$ & $14(0-30)$ \\
\hline
\end{tabular}

a Penicillin (63 events); vancomycin ( 6 events); cephradine ( 4 events); erythromycin ( 3 events).

b Values given as median with range in brackets.

tinuation of this prophylaxis. During streptococcal prophylaxis, fever lead to treatment with broad-spectrum antibiotics, mostly vancomycin, in 25 cases. All patients recovered and fever resolved within a median of three days (range 137 days).

Significantly more febrile episodes in patients receiving streptococcal prophylaxis were classified as fever of unknown origin $(p<0.02)$. Clinically documented infections, both major and minor, were equally distributed among the three groups. The incidence of microbiologically documented infections with positive blood cultures was significantly lower in the streptococcal prophylaxis group compared to both other groups. In only $12 \%$ of febrile episodes in patients receiving streptococcal prophylaxis, was a positive blood culture found compared with $36 \%$ and $30 \%$ in patients receiving ciprofloxacin alone and polymyxin $B$ plus nalidixic acid or neomycin respectively $(\mathrm{p}<0.01)$.

In patients receiving streptococcal prophylaxis, only two episodes of streptococcal septicaemia occurred. In the first case Streptococcus viridans was found in a blood culture from a patient who received erythromycin as alternative prophylaxis because of allergy to penicillin. The isolated strain showed no resistance to erythromycin. In the second case Streptococcus mitis was isolated from a blood culture taken one day after discon- tinuation of prophylaxis in a patient who was still severely neutropenic. In both cases antibiotic treatment with vancomycin was followed by complete and uneventful recovery within 4 to 6 days. In patients receiving ciprofloxacin alone, eight episodes of streptococcal septicaemia were documented $(\mathrm{p}<0.01)$. Four patients recovered within 6 to 22 days. The remaining four patients died of respiratory insufficiency due to adult respiratory distress syndrome. Twenty-one episodes of streptococcal septicaemia occurred in patients receiving polymyxin $B$ plus nalidixic acid or neomycin $(\mathrm{p}<0.001$ ). In 17 cases the median duration of symptoms was six days (range 3-18 days). Four patients developed pulmonary symptoms, two of whom eventually recovered while the remaining two patients died of respiratory insufficiency. In addition, two patients in this prophylaxis group died of Staphylococcus aureus septicaemia.

Colonization. Results of surveillance cultures were compared to analyze the impact of streptococcal prophylaxis on the rate of colonization by microorganisms. With regard to Staphylococcus aureus colonization, no difference was found between patients receiving ciprofloxacin with and without streptococcal prophylaxis ( $8 \%$ and $6 \%$ of treatment events respectively). In the ciprofloxacin plus streptococcal prophylaxis group, aerobic gram-negative bacteria were isolated in surveillance cultures in $18 \%$ of treatment events. 
Table 2: Classification of febrile episodes and infection-related mortality.

\begin{tabular}{|c|c|c|c|}
\hline & $\begin{array}{l}\text { Polymyxin } B+ \\
\text { neomycin or } \\
\text { nalidixic acid }\end{array}$ & Ciprofloxacin & $\begin{array}{c}\text { Ciprofloxacin } \\
\text { streptococcal } \\
\text { prophylaxis }\end{array}$ \\
\hline No. of febrile episodes & 108 & 36 & 65 \\
\hline No. of documented infections & $63(58 \%)$ & $20(56 \%)$ & $26(41 \%)$ \\
\hline No. microbiologically documented & 41 & 14 & 11 \\
\hline Positive blood culture & $32(30 \%)$ & $13(36 \%)$ & $8(12 \%)$ \\
\hline Streptococci & 21 & 8 & 2 \\
\hline Staphylococcus aureus & 6 & 0 & 0 \\
\hline Staphylococcus epidermidis ${ }^{\mathrm{a}}$ & 3 & 4 & 4 \\
\hline Corynebacterium $J K$ & 0 & 1 & 0 \\
\hline Corynebacterium xerosis & 0 & 0 & 1 \\
\hline Pseudomonas maltophilia & 0 & 1 & 0 \\
\hline Klebsiella pneumoniae & 4 & 0 & 0 \\
\hline Branhamella catarrhalis & 1 & 0 & 0 \\
\hline Bacteroides oralis & 0 & 0 & 1 \\
\hline Positive culture at other site & 9 & 1 & 3 \\
\hline Streptococci & 2 & 0 & 0 \\
\hline Staphylococcus aureus & 3 & 0 & 1 \\
\hline Escherichia coli & 1 & 0 & 0 \\
\hline Pseudomonas aeruginosa & 1 & 0 & 0 \\
\hline Pseudomonas maltophilia & 0 & 0 & 1 \\
\hline Klebsiella pneumoniae & 1 & 0 & 0 \\
\hline Bacteroides distasonis & 1 & 0 & 0 \\
\hline Aspergillus fumigatus & 0 & 1 & 1 \\
\hline No. clinically documented & $22(20 \%)$ & $6(17 \%)$ & $15(23 \%)$ \\
\hline Major infection & 5 & 1 & 2 \\
\hline Minor infection & 17 & 5 & 13 \\
\hline No. with fever of unknown origin & $45(42 \%)$ & $16(44 \%)$ & $39(59 \%)$ \\
\hline Infection-related mortality & 4 & 4 & 0 \\
\hline
\end{tabular}

${ }^{a}$ Combined with streptococci: 2 cases on polymyxin $\mathrm{B}+$ nalidixic acid or neomycin, 1 case on ciprofloxacin.

${ }^{b}$ Combined with $S$. epidermidis.

These organisms included Escherichia coli $(\mathrm{n}=1)$, Klebsiella sp. $(\mathrm{n}=1)$, Enterobacter spp. $(\mathrm{n}=3)$, Pseudomonas aeruginosa $(\mathrm{n}=1)$, Pseudomonas spp. $(\mathrm{n}=6)$ and Acinetobacter spp. $(\mathrm{n}=3)$. Only six microorganisms (Pseudomonas spp., $\mathrm{n}=3$ and Acinetobacter spp., $\mathrm{n}=3$ ) were resistant to ciprofloxacin. In two cases polymyxin B was added to the prophylactic regimen. All colonizations were transient. No episodes of colonization with aerobic gram-negative bacteria were found in the group receiving ciprofloxacin without streptococcal prophylaxis $(\mathrm{p}<0.02)$. In patients receiving polymyxin $\mathrm{B}$ plus neomycin or nalidixic acid, significantly more episodes of colonization, both with Staphylococcus aureus (26\%) and aerobic gram-negative bacteria $(51 \%)$, were found compared with patients receiving cipro- floxacin ( $p<0.001$ ). All groups had comparable numbers of episodes of colonization with yeasts and fungi (polymyxin B plus neomycin or nalidixic acid group $55 \%$, ciprofloxacin group $57 \%$ and ciprofloxacin plus streptococcal prophylaxis group $45 \%$ ).

\section{Discussion}

During the past few years, the pathogen distribution of bacterial infection in leukaemic patients undergoing cytotoxic chemotherapy has been changing from gram-negative to gram-positive bacteria. An increased incidence of infections caused by Staphylococcus epidermidis is as- 
sociated with the more frequent use of indwelling central venous catheters $(15,16)$. The increasing incidence of streptococcal septicaemia in patients with leukaemia coincides with the use of more aggressive cytotoxic chemotherapy causing more severe and prolonged neutropenia and a greater degree of mucositis, thereby providing portals of entry for these organisms $(1,2,14)$. Furthermore, the institution of selective intestinal decontamination (4), especially with the newer quinolones $(6,10)$ may play a role.

In our hospital, an increasing incidence of streptococcal septicaemia lead to the administration of penicillin $G$ in addition to ciprofloxacin for prophylaxis in patients receiving cytotoxic chemotherapy for acute myeloid leukaemia. This regimen was only given during the first 14 days following chemotherapy since, according to a previous study (4), the vast majority of cases of streptococcal septicaemia occur during this period when bone marrow suppression and mucositis are maximal.

This prophylaxis resulted in a significantly shorter duration of fever and antimicrobial therapy in patients receiving the regimen compared with patients receiving ciprofloxacin alone $(p<0.001)$ or a combination of polymixin $B$ with nalidixic acid or neomycin ( $\mathrm{p}<0.001$ ). Moreover, in patients receiving penicillin $G$, fever was associated significantly less frequently with positive blood cultures $(\mathrm{p}<0.01)$ compared with patients not receiving streptococcal prophylaxis, whereas significantly more episodes of fever were of unknown origin $(p<0.02)$. The increased incidence of fever of unknown origin can be explained by a rise in the episodes of drug fever caused by penicillin G. Penicillin G may furthermore prevent bacterial growth in blood cultures, thus also contributing to the higher number of episodes of fever of unknown origin.

More importantly, the penicillin $G$ prophylaxis resulted in a significantly decreased incidence of streptococcal septicaemia. Only two episodes of septicaemia caused by streptococci were documented after penicillin $G$ was added to the prophylactic regimen ( 76 treatment events). One case involved the isolation of an erythromycin sensitive streptococcus in a patient who received erythromycin as alternative streptococcal prophylaxis because of allergy to penicillin. Apparently, because erythromycin is bacteriostatic rather than bactericidal, it could not prevent septicaemia. The second case occurred one day after discontinuation of prophylaxis in a still severely neutropenic patient. Both patients recovered uneventfully after antibiotic treatment with vancomycin. Streptococcal septicaemia was documented in 8 of 38 treatment events in patients receiving ciprofloxacin alone $(p<0.01)$, and in 21 of 101 treatment events in patients receiving polymyxin $B$ plus nalidixic acid or neomycin $(\mathrm{p}<0.001)$, with a fatal outcome in four and two cases respectively.

Guiot et al. (12) also reported a decreased incidence of streptococcal infection after the introduction of penicillin $\mathrm{G}$ for prophylaxis. In another study, Guiot et al. (17) demonstrated that infections with viridans streptococci occurred less frequently and were less severe in patients on penicillin $G$ prophylaxis compared with patients on cotrimoxazole prophylaxis.

A potential disadvantage of penicillin $G$ prophylaxis is overgrowth of aerobic gram-negative bacteria as well as yeasts and fungi. However, although gram-negative bacteria were isolated in surveillance cultures significantly more frequently in patients receiving ciprofloxacin plus penicillin $G$ compared with patients receiving ciprofloxacin alone $(p<0.02)$, this colonization did not lead to infection. In our study, administration of penicillin $\mathrm{G}$ did not result in overgrowth of yeasts and fungi.

Another potential disadvantage is the emergence of penicillin-resistant streptococci (18) or Staphylococcus epidermidis (19). Therefore, it was our policy to replace penicillin $G$ with vancomycin on an empirical basis in case of fever. However, infections with penicillin-resistant streptococci were not documented in our study. Furthermore, an increased incidence in septicaemia caused by Staphylococcus epidermidis was not found in patients receiving penicillin $\mathrm{G}$ (Table 2).

In conclusion, a prophylaxis regimen consisting of penicillin $\mathrm{G}$ and ciprofloxacin proved to be highly successful in preventing infections caused by streptococci and in reducing infection-related mortality. It remains unclear which alternative prophylaxis is preferable in the case of penicillin allergy. Erythromycin does not seem to be the most appropriate choice. Vancomycin may be a better alternative.

\section{References}

1. Cohen J, Worsley AM, Goldman IM, Donnelly JP, Catorsky D, Galton DAG: Septicaemia caused by viridans streptocci in neutropenic patients with leukaemia. Lancet 1983, ii: 1452-1454. 
2. Kern W, Kurrle E, Vanek E: High risk of streptococcal septicemia after high dose cytosine arabinoside treatment for acute myelogenous leukemia. Klinische Wochenschrift 1987, 65: 773-780.

3. Menichetti F, Del Favero A, Guerciolini R, Tonato M, Frongillo RF, Roila F, Pauluzzi S: Viridans streptococci septicemia in cancer patients: a clinical study. European Journal of Epidemiology 1987, 3: 316-318.

4. Van der Lelie H, van Ketel RJ, von dem Borne AEGK, van Oers RHJ, Thomas BLM, Goudsmit R: Incidence and clinical epidemiology of streptococcal septicemia during treatment of acute myeloid leukemia. Scandinavian Journal of Infectious Diseases 1991, 23: 163168.

5. Dybedal I, Lamvik J: Respiratory insufficiency in acute leukemia following treatment with cytosine arabinoside and septicemia with Streplococcus viridans. European Journal of Haematology 1989, 42: 405-406.

6. Kern W, Kurrle E, Schmeiser T: Streptococcal bacteremia in adult patients with leukemia undergoing aggressive chemotherapy. A review of 55 cases. Infection 1990, 18: 138-145.

7. McWhinney PHM, Gillespie SH, Kibbler CC, Hofthrand AV, Prentice HG: Streptococcus mitis and ARDS in neutropenic patients. Lancet 1991, 337: 429.

8. Aming M, Gehrt A, Aul C, Runde V, Hadding U, Schneider W: Septicemia due to Streptococcus mitis in neutropenic patients with acute leukemia. Blut 1990, 61: $364-368$.

9. Henslec J, Bostrom B, Weisdorf D, Ramsay $\mathbf{N}$, McGlave P, Kersey J: Streptococcal sepsis in bone marrow transplant patients. Lancet 1984, i: 393.

10. Classen DC, Burke JP, Ford CD, Evershed S, Aloia MR, Wilfahrt JK, Elliott JA: Streptococcus mitis sepsis in bone marrow transplant patients receiving oral antimicrobial prophylaxis. American Journal of Medicine 1990, 89: 441-446.

11. Rozenberg-Arska M, Dekker A, Verdonck L, Verhoef J: Prevention of bacleremia caused by alpha-hemolytic streptococci by roxithromycin (RU-28965) in granulocytopenic patients receiving ciprofloxacin. Infection 1989, 17: 240-244.
12. Guiot HFL, Peters WG, van den Broek PJ, van der Meer, JWM, Kramps JA, Willemze R, van Furth R: Respiratory failure elicited by streptococcal septicacmia in patients treated with cytosine arabinoside, and its prevention by penicillin. Infection 1990, 18: 131-137.

13. Karp JE, Dick JD, Angelopulos C, Charache P, Green L, Burke PJ, Saral R: Empiric use of vancomycin during prolonged treatment-induced granulocytopenia. Randomized, double-blind, placebo-controlled clinical trial in patients with acute leukemia. American Journal of Medicin 1986, 81: 237-242.

14. Heimdahl A, Mattsson T, Dahllöf G, Lönnquist B, Ringden 0: The oral cavity as a port of entry for early infections in patients treated with bone marrow transplantation. Oral Surgery, Oral Medicine, Oral Pathology 1989, 68: 711-716.

15. Wade JC, Schinpff SC, Newman KA, Wiernik PH: Staphylococcus epidermidis: an increasing cause of infection in patients with granulocytopenia. Annals of Internal Medicine 1982, 97: 503-508.

16. Rubin M, Hathorn JW, Marshall D, Gress J, Steinberg SM, Pizzo PA: Gram-positive infections and the use of vancomycin in 550 episodes of fever and neutropenia. Annals of Internal Medicine 1988, 108: 30-35.

17. Guiot HFL, van der Meer JWM, van den Brock PJ, Willemze R, van Furth R: Prevention of viridans-group streptococccal septicemia in oncohematologic patients: a controlled comparative study on the effect of penicillin $\mathrm{G}$ and cotrimoxazole. Annals of Hematology 1992, 64: 260-265.

18. Santini $\mathbf{C}$, Venditti $\mathbf{M}$, Baiocchi P, Brandimarte $\mathbf{C}$, Serra P, Mandelli F, Girmenia C, Micozzi A, Martino P: Emergence of penicillin-resistant viridans streptococci causing septicemia in granulocytopenic patients. European Journal of Epidemiology 1988, 4: 391-392.

19. Van de Leur JJJPM, Dotferhoff ASM, van Turnhout JM, Vollaard JE, Clasener HAL: Colonisation of oropharynx with staphylococci after penicillin in neutropenic patients. Lancet 1992, 340: 861-862. 